\title{
AS TECNOLOGIAS NA MEDICINA 4.0 E A RESISTÊNCIA MÉDICA AOS NOVOS SISTEMAS
}

\section{ARTIGO DE REVISÃo}

FERRAZ, Fábio Henrique de Carvalho ${ }^{1}$

FERRAZ, Fábio Henrique de Carvalho. As tecnologias na medicina 4.0 e a resistência médica aos novos sistemas. Revista Científica Multidisciplinar Núcleo do Conhecimento. Ano. 07, Ed. 02, Vol. 01, pp. 05-18. Fevereiro de 2022. ISSN: 24480959, Link de

acesso: https://www.nucleodoconhecimento.com.br/tecnologia/tecnologias-na$\underline{\text { medicina }}$

\section{RESUMO}

A influência do avanço tecnológico, principalmente no setor da saúde, tem impactado de modo positivo a medicina e suas práticas interventivas uma vez que, a todo momento, surgem novas técnicas e métodos capazes de melhorar o diagnóstico e o tratamento de inúmeras doenças. Nos últimos anos, a medicina, junto aos programas ligados a tecnologia da informação e inteligência artificial, tem promovido verdadeiras transformações com a criação de, por exemplo, projetos de sensores inteligentes e algoritmos robóticos que asseguram conforto e segurança ao paciente podendo ser monitorado de sua própria residência. Nesse diapasão, a combinação de conhecimentos médicos, aliados aos princípios e práticas da engenharia, formam a saúde/medicina 4.0. Cumpre informar que essa logística possibilita maiores chances de sucesso na prevenção, bem como na cura de inúmeras doenças. Contudo, a sociedade médica atual vive um dilema com profissionais pouco capacitados devido

1 Pós-graduado em Engenharia de desenvolvimento de projetos eletrônicos e em Engenharia de Automação e Eletrônica Industrial.

RC: 106092

Disponível em: https://www.nucleodoconhecimento.com.br/tecnologia/tecnologias-namedicina 
à resistência que os próprios apresentam para atuarem com novas tecnologias, e, numa perspectiva multidisciplinar, somente o conhecimento médico não é suficiente para acompanhar a saúde 4.0. Neste contexto, visamos responder: Como atenuar a falta de capacitação e resistência médica para que possam atuar corretamente com as novas demandas tecnológicas no setor da saúde? Tendo como objetivo elencar os principais problemas relatados pela experiência dos próprios profissionais médicos de acordo com a literatura publicada. Com isso, a partir de pesquisas bibliográficas, este artigo propõe apresentar um pouco da evolução tecnológica sofrida no setor da engenharia impactando diretamente o setor da saúde e de que forma tal evolução repercutiu nas atividades médicas. Além disso, pretende-se apresentar uma possível solução aos problemas relatados. Os fatos mostram que, em suma, os benefícios dos avanços tecnológicos voltados a medicina 4.0 são reconhecidamente eficazes e que investir em treinamentos para capacitação de profissionais na área de saúde é um caminho, entretanto, as organizações que desejam estes sistemas precisam estar conscientes de que o processo de implantação pode ser uma tarefa difícil que exige a capacidade de suportar a influência de diversos fatores, onde o principal deles é a resistência humana a novas tecnologias.

Palavras-Chave: Evolução Tecnológica, Projetos Eletrônicos, Saúde 4.0, Inteligência Artificial, Resistência Médica.

\section{INTRODUÇÃO}

$\mathrm{Na}$ perspectiva da evolução tecnológica a partir do desenvolvimento de projetos voltados para a área médica, constata-se que tais projetos têm, a cada dia, contribuído de forma eficaz para o desenvolvimento humano. Nesse contexto, destacam-se: Inteligência Artificial, óculos digitais, hologramas, impressão 3D, internet das coisas, big data etc.; que são as principais ferramentas inovadoras que contribuem, principalmente, para tecnologias aplicadas a saúde 4.0. Isso gera forte impacto na forma como novos tratamentos são desenvolvidos na gestão da saúde e até mesmo no acompanhamento de pacientes. Assim, com o uso de sistemas computadorizados,

RC: 106092

Disponível em: https://www.nucleodoconhecimento.com.br/tecnologia/tecnologias-namedicina 
os profissionais da área de engenharia buscam desenvolver novas tecnologias para as práticas voltadas à atuação médica proporcionando a cura de diversas doenças, antes indecifráveis para a medicina (CALDAS, 2021). Com isso, é importante que estes profissionais possam atuar em conjunto com os profissionais médicos, havendo troca de conhecimento, resultando em mecanismos e instrumentos que sejam cada vez mais velozes e precisos para o cotidiano hospitalar. Sendo assim, a fusão dos conhecimentos e a densa união dos profissionais de ambas as áreas possibilitará muitos outros benefícios para toda a coletividade sendo possível, por exemplo, intervenções cirúrgicas de alta complexidade ocorrendo de forma segura e eficaz (VERZUH, 2000).

Já é perceptível a chegada das inovações em todas as especialidades médicas, principalmente no ramo cirúrgico (cardiologia, cerebral etc.), beneficiando pacientes e médicos através da precisão e velocidade da informação tão necessária para a salvação de vidas. Por isso, pode-se dizer que a expectativa de vida de uma sociedade está diretamente ligada às tecnologias implantadas na área médica e na capacitação de seu pessoal, proporcionando diagnósticos cada vez mais assertivos que desacelerem a progressão ofensiva de várias doenças. É interessante o jeito como se sentem os profissionais de engenharia, atuantes na tecnologia médica, com seus feitos e dons, os quais beneficiam o bem mais precioso, a saber, a vida humana (MURARO, 2009).

Apesar dessa perspectiva tecnológica em benefício da medicina 4.0, a sociedade médica atual ainda sofre com profissionais não capacitados e outros que ainda resistem a alternativas tecnológicas na rotina médica. Neste contexto, visamos responder: Como atenuar a falta de capacitação e resistência médica para que possam atuar corretamente com as novas demandas tecnológicas no setor da saúde? Tendo como objetivo elencar os principais problemas relatados pela experiência dos próprios profissionais médicos de acordo com a literatura publicada. Não basta apenas a contribuição da engenharia criando projetos inovadores, sobretudo, é

RC: 106092

Disponível em: https://www.nucleodoconhecimento.com.br/tecnologia/tecnologias-namedicina 
preciso também agregar um conjunto de alternativas que torne os profissionais e toda a equipe médica receptiva aos conhecimentos das novas demandas criadas, além claro dos conhecimentos por eles já adquiridos na sua formação. Dentro desse contexto, o presente artigo foi elaborado de modo a apresentar os avanços na saúde 4.0 e averiguar quais são as principais causas para a resistência médica à implantação de sistemas tecnológicos, principalmente no que tange a parte de registros eletrônicos que é onde se encontra maior resistência por parte dos profissionais médicos. Para alcançar este objetivo, a pesquisa bibliográfica será a principal ferramenta.

\section{DESENVOLVIMENTO}

\subsection{O PAPEL DA ENGENHARIA NA ÁREA MÉDICA E OS DESAFIOS ATUAIS}

Sabe-se que a engenharia voltada para a criação de projetos eletrônicos na área médica tem atuação forte nas clínicas e em grandes centros hospitalares. Dessa forma, essa prática busca manter uma correta estrutura para a área da saúde através da implantação de diversos instrumentos e processos, os quais beneficiam a medicina (BARRA, 2010). Todos os métodos utilizados nas ciências matemáticas são realizados nesse âmbito, formando verdadeiros sistemas biológicos modernos. De fato, foi nesse momento que a área médica se viu restringida em seus esforços e considerou a necessidade de junção com a área tecnológica fazendo com que a inovação contribuísse com a salvação e prevenção de várias doenças. Os fatores são explicados pelo fato de que a eletromedicina usa as ferramentas matemáticas, bem como os processos físicos e químicos, a fim de estabelecer uma ligação com as funções biológicas, criando teorias acerca do entendimento do corpo humano, de modo a usar técnicas e metodologias especiais (ANTUNES, 2010). Assim, foram criados órgãos artificiais como base para um estudo eficaz, além de importantes equipamentos relacionados a implantes, dentre outras práticas. $O$ resultado dessa

RC: 106092

Disponível em: https://www.nucleodoconhecimento.com.br/tecnologia/tecnologias-namedicina 
junção foi o crescimento de grandes centros hospitalares e importantes sistemas e projetos criados para o combate e prevenção dos diversos tipos de doenças.

Entretanto, sabe-se que, quando o assunto é o organismo humano, os desafios são intensos. Desse modo, as práticas de engenharia relacionadas à projetos eletrônicos na área da medicina têm evoluído de acordo com o crescimento da consciência social. A dedicação à pesquisa deve ser constante, pois apesar dos intensos esforços, muitas especialidades médicas necessitam de implementações novas. Uma das áreas mais beneficiadas está ligada à ortopedia, além da especialidade cardiorrespiratória, uma vez que ela possui importantes elementos disponíveis para a pesquisa. De fato, afirmou Infantosi (2001):

O que atualmente se entende como bioengenharia está muito ligado ao desenvolvimento da instrumentação e, em particular, à instrumentação médica, cujo desenvolvimento é necessário a interação de médicos e engenheiros, nestes incluídos físicos, químicos, matemáticos e cientistas da computação. Surgem então outros campos ligados a bioengenharia, como bioinformática, genômica etc.

A criação da engenharia biomédica, de fato ocorreu, devido ao empenho de engenheiros decididos a investir na área, buscando tecnologia considerada de ponta, a fim de resolver vários problemas na área da saúde. De fato, não somente a medicina, mas vários setores da ciência biológica tiveram extraordinários ganhos. Dessa forma, sabe-se que o sucesso ocorrido na área médica e biológica é resultado de vários conhecimentos contidos na matemática, física, engenharia, tecnologia da informação, dentre outras áreas do conhecimento, relacionado às exatas. Destarte, a união de várias disciplinas fez com que a área médica tivesse sucesso e passasse a atuar com modernas tecnologias.

Conforme referenciado anteriormente, para que haja uma verdadeira interação entre os profissionais das mais variadas áreas, a fim de ocorrer o benefício para a área médica, muitas atitudes devem ser deixadas de lado, tais como o orgulho e a vaidade, uma vez que todos os profissionais (físicos, engenheiros, matemáticos e médicos)

RC: 106092

Disponível em: https://www.nucleodoconhecimento.com.br/tecnologia/tecnologias-namedicina 
devem atuar em consonância e respeito para um bem maior. Assim, quando isso ocorrer, o sucesso realmente é garantido (PAIM, 2005). Sendo assim, os profissionais que atuam nesse sentido, o fazem com paixão pela profissão e visão do bem-estar de uma sociedade. Acerca dessa questão afirmou Oliveira (2003):

O que por um lado, historicamente se justifica hoje em dia, já não deveria mais acontecer, porque a engenharia biomédica/bioengenharia não utiliza somente conceitos da engenharia elétrica. Utiliza-se também das outras engenharias, por exemplo, da mecânica. Então, o que acontece, às vezes, até mesmo o fato de um grupo de Engenharia/ Bioengenharia estar inserido dentro do departamento sobre a inter e multidisciplinariedade da Engenharia Biomédica/ Bioengenharia, pode ser um fator limitante. Isso pode acarretar uma inibição na criação de linhas de pesquisa com menor ênfase. Enfim, essa situação, às vezes resulta na não criação de uma nova linha por se estar umbilicalmente ligada a um departamento de engenharia elétrica. Ou então, em uma mesma universidade trabalhando em separado, quando eles deveriam estar trabalhando em conjunto.

Assim, dentre as atividades de tal profissional, encontrar-se-ão pesquisas na área de engenharia celular, próteses biomecânicas, engenharia cardiovascular, bem como relacionados ao sistema respiratório, dentre tantas outras atuações que têm beneficiado a saúde e a cura das doenças no mundo. Nesse sentido, ainda observou Oliveira (2003):

Os equipamentos, em particular os digitais, foram cada vez mais incorporados à rotina das unidades de atendimento à saúde, em especial às hospitalares. $E$ isso gerou, inicialmente, uma nova necessidade: o gerenciamento dos equipamentos. Até então, o gestor dessas unidades era profissional da área médica; $A$ incorporação da tecnologia cada vez mais sofisticada e em maior número, se por um lado, contribui para a melhoria da qualidade do atendimento à saúde, poderia aumentar, em contrapartida, os custos deste atendimento. Logo, tornou-se necessário formar equipes constituídas de especialistas na gestão desta tecnologia; surge, então, a engenharia clínica. No Brasil e demais países, o impacto das novas tecnologias hospitalares, bem como a revolução tecnológica da década de 80 , mostrou a importância de se mostrar tais profissionais. Desta forma, a atuação do profissional da Engenharia Biomédica/Bioengenharia passa a se dar também dentro do sistema de saúde, no qual ele passa a ser consultado tanto sobre a aquisição do equipamento quanto em

RC: 106092

Disponível em: https://www.nucleodoconhecimento.com.br/tecnologia/tecnologias-namedicina 
relação ao seu uso mais adequado. A engenharia clínica surge, por conseguinte, como uma consequência da incorporação da tecnologia no atendimento à saúde, e o profissional que exerce esta função é um engenheiro biomédico com atuação neste ramo.

Com isso, a bioengenharia tornou-se uma verdadeira necessidade na melhoria dos serviços relacionados à saúde. No tocante ao crescimento da tecnologia no Brasil, sabe-se que foi, principalmente após 1990, que ocorreu o crescimento de cursos de pós-graduação nas áreas de engenharia biomédica, onde houve uma real estruturação do tema. Entretanto, muitas restrições e barreiras acontecem no cotidiano (COPPE/UFRJ, 2021):

\begin{abstract}
Apesar do contexto desfavorável à expressão da criatividade que resulta em desenvolvimento de tecnologia e inovação, os professores e alunos do Programa de Engenharia Biomédica da Coppe não desistiram. Principalmente na área de Instrumentação Biomédica, a pioneira do Programa, continuaram a estudar e desenvolver sistemas e equipamentos médicos, em teses e dissertações que persistem na disposição de contribuir para gerar tecnologia médica adaptada à realidade brasileira, em termos de custos e de acessibilidade. Por ter seu foco no desenvolvimento de instrumentação para a área da saúde em geral, a área de Instrumentação atua numa variada gama de interesses, em linhas de pesquisa que vão mudando ao longo do tempo. Nos anos 1990 havia, por exemplo, uma linha de óptica, que trabalhava no desenvolvimento do uso de laser e fibras ópticas e foi descontinuada com a saída do professor que a comandava. Algumas linhas, porém, permanecem desde os tempos iniciais, como a de bioimpedância, na qual os pesquisadores buscam explorar, em variadas aplicações médicas, o fenômeno biofísico da resistência que os tecidos biológicos oferecem à passagem de correntes elétricas.
\end{abstract}

A procura por engenheiros biomédicos tem crescido a cada dia sendo que, atualmente, são vários cursos de graduação espalhados em vários lugares do país, como por exemplo em: Santo André, São José dos Campos, Montes Claros, Belo Horizonte, Uberlândia, Rio de Janeiro, dentre outros estados (COPPE/UFRJ, 2021). Dessa forma, esses cursos buscaram atender à demanda atual de engenheiros biomédicos, os quais atuam em vários centros de saúde, espalhados pelo país. É importante mencionar a responsabilidade do engenheiro que atua na área de eletromedicina, uma vez que sua formação deve, de fato, ser estruturada, pois tal

RC: 106092

Disponível em: https://www.nucleodoconhecimento.com.br/tecnologia/tecnologias-namedicina 
profissional deve ser um profundo conhecedor de várias disciplinas, principalmente relacionada à anatomia humana. $\mathrm{O}$ conhecimento do engenheiro ainda abrange técnicas de organização e gestão, devendo possuir ampla inteligência emocional para ocorrer uma boa atuação em grandes grupos, liderando de forma eficaz e inovadora, a fim de beneficiar a população. Destaca-se, ainda, a área de engenharia pulmonar, através do estudo da ventilação mecânica. Neste interim, os ventiladores pulmonares começaram a serem controlados de forma eletrônica. Assim, a partir do ano 2000, o estudo foi expandido e passou a ser dirigido para a área da fisiologia do exercício Expohospital Brasil (2021). Esse período pontuou grandes avanços tecnológicos, lembrando que esse fator, poderia impedir a continuidade de tais tecnologias, tanto nos sistemas de saúde públicos, quanto nos demais.

Assim, fez-se urgente a manifestação de gestores da área para que procurassem soluções específicas a respeito do problema. Apesar das barreiras, o futuro da eletromedicina é promissor, uma vez que são desenvolvidos grandes projetos nas áreas de engenharia relacionada à neurologia, além da bioinformática, biomecânica, dentre outras, conforme pode-se averiguar nas normas visualizadas pelo Conselho Regional de Medicina do Estado de São Paulo (CRMSP) em sua resolução 097. As técnicas utilizadas tornam-se a cada dia mais avançadas, sendo que o campo da engenharia neural tem avançado consideravelmente, uma vez recebe apoio de vários setores. Nesse sentido, é fixado um estimulador elétrico no cérebro de pessoas que possuem Parkinson, a fim de melhor observar o funcionamento cerebral (KENSKI, 2011). No mesmo sentido, as premissas observadas pela Coppe/Ufrj (2021), tornaram-se cada vez mais seguras, a saber:

Uma das mais recentes linhas de pesquisa do Programa é a bioinformática. Implementada no Laboratório de Engenharia de Sistemas de Saúde, busca desenvolver modelos matemáticos para analisar o genoma e classificar as informações obtidas. Essas técnicas permitem identificar, por exemplo, padrões de grupos de genes que se associam para provocar uma determinada doença.

RC: 106092

Disponível em: https://www.nucleodoconhecimento.com.br/tecnologia/tecnologias-namedicina 
Portanto, os avanços são notórios e cada vez mais inovadores. Entretanto, os profissionais devem estar atentos para possíveis erros na execução de tais tipos de equipamentos, uma vez que, contata-se diversos tipos de perdas e desperdícios de vários equipamentos, por falta de profissionais capacitados. Nesse sentido, o perfil do engenheiro biomédico deve ser criativo e motivador, uma vez que um profissional eficaz e competente, atuará tanto em hospitais e empresas, na elaboração de novos projetos eletrônicos voltados para a saúde. Outro campo que abre portas para a eletromedicina diz respeito às Universidades e pesquisas acadêmicas, através de grandes centros e Universidades Públicas, bem como instituições privadas (PREGUER, 2005), onde a atuação é realizada em projetos na área da bioengenharia, utilizando instrumentos tais como próteses, órteses, além de órgãos artificiais.

Como mencionado anteriormente a gestão hospitalar é um campo fértil para a atuação do engenheiro hospitalar, de forma a treinar profissionais para a correta manutenção dos aparelhos eletrônicos utilizados reduzindo gastos e perdas desnecessárias, como verifica-se na Expohospital Brasil (2021):

As condições relacionadas ao uso, manutenção e gestão de equipamentos odontomédico hospitalares, de um modo geral, são bastante precárias no país, principalmente pela falta de profissionais especializados na área. Atualmente, devido à ausência de profissionais com formação específica para essa atividade, os hospitais contratam engenheiros civis, engenheiros eletricistas ou, até mesmo, técnicos ou físicos com especialização em física médica para desempenhar as atividades de recuperação, especificação e manutenção de equipamentos.

Assim, toda a estruturação e planejamento dentro de um grande centro passa primeiramente pelo biogenheiro, o qual planeja toda a estrutura relacionada à instalação de equipamentos, averigua os possíveis riscos e aprimora a qualidade do local, o qual deve ser detalhadamente inspecionado sendo que sua prática é desde a estrutura elétrica e hidráulica do centro hospitalar, até as práticas relacionadas a uma eficiente gestão, tornando-se, um desafio atual na eletromedicina.

RC: 106092

Disponível em: https://www.nucleodoconhecimento.com.br/tecnologia/tecnologias-namedicina 
Somada a todas essas informações, referentes às novas demandas da engenharia médica, atualmente, a medicina tem sido objeto de constantes estudos e aprofundamentos concernentes aos novos projetos eletrônicos, os quais usam diferentes possibilidades e recursos, a fim de fornecer qualidade de vida para pacientes e médicos, culminando na chamada medicina 4.0, a qual tem por desafio unir cada vez mais profissionais da saúde, com os profissionais que atuam no desenvolvimento de projetos eletrônicos (KENSKI, 2011). Somente com o conhecimento multidisciplinar é possível utilizar a inteligência artificial na contribuição de práticas terapêuticas, a saber, prevenções, diagnósticos, tratamentos em unidades intensivas, tratamentos feitos por telemedicina, dentre outras possibilidades, as quais culminam para o avanço da medicina.

\subsection{OS AVANÇOS TECNOLÓGICOS NA SAÚDE E $O$ COMPORTAMENTO MÉDICO À IMPLANTAÇÃO DE NOVAS TECNOLOGIAS}

A demanda por novas tecnologias aumentou no ano de 2020 com as consequências trazidas pela Pandemia da COVID-19 fazendo com que importantes países da Europa, a exemplo da Itália, experimentassem sobrecargas em seus respectivos sistemas de saúde e evidenciassem a necessidade de se investir em suas instalações com tecnologias e modelos de atendimento à saúde (FREDERICO, 2021). É inegável, assim, que as questões tecnológicas na pandemia foram barreiras encontradas em importantes países considerados desenvolvidos.

As verificações de financiamentos corretos dentro de um sistema satisfatório de gestão são pilares para que as tecnologias médicas sejam colocadas em prática em qualquer lugar do mundo (LOBO, 2021). Especificamente no Brasil, o autor supramencionado afirmou que os avanços têm ocorrido de forma positiva, uma vez que os armazenamentos em nuvem destacaram as técnicas trazidas pela inteligência

RC: 106092

Disponível em: https://www.nucleodoconhecimento.com.br/tecnologia/tecnologias-namedicina 
artificial fazendo com que a medicina tenha resultados de exames certeiros e chances de maiores curas de importantes e devastadores doenças.

Dentre os avanços, a inteligência artificial (IA) tornou-se destaque na engenharia eletromédica, uma vez que, especificamente na área da saúde, a IA possibilita diminuição de mortes, prevenções e menor incidência de erros médicos, como verificado por Lobo (2021):

\begin{abstract}
Há que se considerar que a identificação de problemas e padrões ("pattern recognition") pelo computador, reconhecendo, por exemplo, uma lesão dermatológica, ou fazendo um laudo em um exame de imagem, ou, ainda, pelo processamento de um grande volume de dados de pacientes ("big data"), poderá indicar o "know what" de um problema de saúde. Mas, caberá ao médico discutir o caso com seu paciente agregando o seu "know-why", orientando-o e aliviando suas tensões, já que o computador não tem emoções e uma compreensão do "outro".
\end{abstract}

Com isso, começa-se a perceber que, com a chegada de novas demandas tecnológicas, o nível de exigência quanto a capacitação e interação entre médico e paciente acaba sendo maior também, sendo que o primeiro deve estar atento às inovações constantes, estendendo maior autonomia ao paciente, o qual também deverá engajar-se nas novas interações trazidas pela telemedicina.

Uma outra questão pertinente que também vem afetando a forma como os profissionais de saúde trabalham, é o uso da tecnologia da informação para remodelagem das práticas médicas, que tratam de aplicações voltadas a processos administrativos e procedimentos clínicos. Basicamente, nos últimos anos, as organizações hospitalares buscaram aproveitar as oportunidades geradas pelo avanço na tecnologia com o objetivo de melhorar a qualidade dos serviços prestados, reduzir custos operacionais e aumentar a eficiência dos processos administrativos e clínicos. No entanto, algumas pesquisas têm sinalizado para o fato de que vários hospitais têm consumido grandes quantidades de recursos financeiros devido a iniciativas malsucedidas (PARÉ, 2002). Já outros estudos reconheceram que a resistência do médico é uma das principais causas para a subutilização ou fracasso RC: 106092

Disponível em: https://www.nucleodoconhecimento.com.br/tecnologia/tecnologias-namedicina 
de tais implementações tecnológicas (LAPOINT et al., 2002; TAN, 2005; HORAN et al., 2005).

Segundo Horan et al. (2005), os médicos formam um grupo de usuários com características distintas e diferenciadas dos demais usuários de computadores, sendo altamente pressionados pelo tempo e lidando com informações e decisões vitais. Com isso, tornam-se um desafiante grupo para a aceitação de novas tecnologias. Adentrando nas principais causas (problemas) que geram a resistência médica à implantação de novos sistemas citada no trabalho de Magalhães (2006), temos: Ausência de segurança, flexibilidade do sistema, infraestrutura tecnológica, falta de familiaridade dos médicos com o uso de computadores.

- Ausência de Segurança

Alguns médicos indagam quanto a autenticidade e validade jurídica de prescrições feitas eletronicamente. A questão da segurança e privacidade dos dados do paciente é, sem dúvidas, extremamente importante para a prescrição eletrônica. O temor de que a ação dos hackers e a insegurança do sistema possam levar a perda de informações médicas assustam a maioria dos usuários (CHEONG, 1996; MAGALHÃES, 2006).

- Flexibilidade do Sistema

É alegada falta de flexibilidade do sistema para realizar determinadas tarefas, tais como: dificuldades de inserir medicações no sistema e refazimento de toda a prescrição caso algum item precise ser acrescentado em uma determinada sequência. Essa questão pode estar relacionada à falta de participação dos médicos no processo de elaboração do desenho do sistema. Por essa razão, o sistema se mostra pouco prático e ineficiente para a realização das tarefas do médico (MAGALHÃES, 2006).

- Infraestrutura Tecnológica

RC: 106092

Disponível em: https://www.nucleodoconhecimento.com.br/tecnologia/tecnologias-namedicina 
De acordo com a percepção de alguns médicos, o sistema em si, em alguns momentos, torna-se lento e instável. O tempo de resposta do sistema mais somado ao esforço despendido na entrada de dados representa, para os médicos, desperdício de tempo (MAGALHÃES, 2006).

- Falta de familiaridade dos médicos com o uso de computadores

Normalmente, todo o sistema é projetado para ser simples e de fácil aprendizado. Contudo, a falta de conhecimento e familiaridade no uso de computadores faz com que alguns médicos tenham dificuldades em utilizar o sistema, principalmente para médicos mais idosos que alegam não ver benefício no sistema (MAGALHÃES, 2006).

Perante os problemas mencionados anteriormente voltamos a questão inicial da problemática: Como atenuar a falta de capacitação e resistência médica para que possam atuar corretamente com as novas demandas tecnológicas no setor da saúde? E a resposta está na criação de um sistema mais simples e de fácil aprendizado, além da capacitação instrumental através de treinamento para que os médicos usem de forma correta o sistema. Por conseguinte, essa ausência de educação tecnológica serviu de barreira para o processo de implantação. Esse fato sugere que sem a necessária educação e treinamento do médico, as dificuldades para a utilização do sistema podem frustrar toda uma tentativa de informatização (MUNDY, 2004).

\section{CONSIDERAÇÕES FINAIS E CONCLUSÕES}

Diante dos fatos, constata-se que a inovação tecnológica na saúde é fator cada vez mais presente na realidade atual e o avanço na área médica tem ocorrido, aumentando assim a expectativa de vida da população. Fatos novos, como o surgimento de robôs, têm feito com que tecnologias de ponta surjam, com a devida união entre a área médica e a engenharia. Como consequência, os diagnósticos tornam-se cada vez mais assertivos.

RC: 106092

Disponível em: https://www.nucleodoconhecimento.com.br/tecnologia/tecnologias-namedicina 
Conforme analisado, a pesquisa e o desenvolvimento são palavras chaves para o sucesso na área. As barreiras empresariais e os interesses pessoais precisam ser quebrados para que de fato, a excelência na área da saúde aconteça e beneficie a todos, e não simplesmente o interesse de uma minoria. Portanto, o profissional médico que atua de forma a buscar a cura das pessoas deve reconhecer a necessidade da engenharia na área da saúde. Além disso, é importante que o governo crie políticas facilitadoras para a junção das áreas biológicas com as exatas, uma vez que, na medicina atual, já se fala em inteligência artificial como uma ferramenta auxiliar na coleta de dados médicos e análises de prontuários contribuindo com uma melhor organização clínica e hospitalar.

Já quanto a questão da falta de capacitação e resistência médica existente as novas tecnologias, problemática levantada neste estudo, principalmente as voltadas a área de tecnologia da informação, é importante saber exatamente o contexto onde o sistema será implantado, de modo a compreender claramente como ele será percebido pelos seus potenciais usuários (FURNIVAL, 1995), levando em consideração que a criação de um sistema mais simples e de fácil aprendizado, além da capacitação instrumental, através de treinamento para os médicos é um dos caminhos a serem seguidos para atenuar essa questão. Em suma, já é fato que os benefícios dos sistemas voltados a tecnologia da informação na área médica são reconhecidamente superiores aos métodos tradicionais. Sendo assim, o método eletrônico oferece um melhor e mais ágil canal de comunicação entre médicos e outros profissionais de saúde, reduz os erros de medicação, transcrição e os custos relacionados ao manuseio e arquivamento de papel, entre outros. Atualmente, para maior motivação dos usuários médicos, as aplicações a partir de novas tecnologias têm visado a simulação de situações reais, aplicado ao treinamento de profissionais para reduzir, ou até mesmo eliminar a resistência apresentada por parte dos médicos que já tem certa idade e não se familiarizaram com sistemas computadorizados.

RC: 106092

Disponível em: https://www.nucleodoconhecimento.com.br/tecnologia/tecnologias-namedicina 


\section{REFERÊNCIAS}

A Evolução da Engenharia Biomédica e os Avanços para o Setor de Saúde. Disponível em: <http://expohospitalbrasil.com.br/a-evolucao-da-engenhariabiomedica-e-os-avancos-para-o-setor-de-saude/>. Acesso em 05 de dezembro de 2021.

ANTUNES, E. et al. Gestão da tecnologia biomédica: tecnovigilância e engenharia clínica. Paris: Editions Scientifiques ACODESS, 2010. 210 p.

BARRA, DAL SASSO. Tecnologia Móvel À Beira Do Leito: Processo de Enfermagem Informatizado Em Terapia Intensiva A Partir Da Cipe 1.0® Texto Contexto Enferm. 2010 JAN-MAR. 19(1):54-63.

CALDAS, R.A. A Construção de um arcabouço legal para Ciência, Tecnologia e Informação. Revista de Ciência Tecnologia e Informação. Disponível em: <http://www.inovacao.uema.br/imagensnoticias/files/A\%20construcao\%20de\%20um \%20modelo\%20de\%20arcabouco\%20legal\%20para\%20Ciencia,\%20Tecnologia\%20 e\%20Inovacao.pdf.> Acesso em: 07 de novembro de 2021.

CHEONG IR. Privacy and security of personal health information. Journal of Informatics in Primary Care 1996: 15-17.

CONSELHO REGIONAL DE MEDICINA DO ESTADO DE SÃO PAULO. Resolução no 097/2001. Institui o Manual de Princípios Éticos para Sites de Medicina e Saúde. Diário Oficial do Estado de São Paulo, 9 de novembro de 2001.

DANIELLI, O. Saúde 4.0: A revolução Entre Conexão e Inteligência Nos Cuidados na Saúde. Disponível em: <https://blog.neomed.tech/saude-4-0-a-revolucao-entreconexao-e-inteligencia-nos-cuidados-na-saude/>. Acesso em: 04 de novembro de 2021.

RC: 106092

Disponível em: https://www.nucleodoconhecimento.com.br/tecnologia/tecnologias-namedicina 
FRANÇA, GENIVAL VELOSO. Telemedicina. Revista Bioética. Disponível em: $<$ https://revistabioetica.cfm.org.br/index.php/revista_bioetica/article/view/266>. Acesso em: 07 de novembro de 2021.

FREDERICO, LUIS FERNANDO. O que startups de telemedicina e healthtechs podem ensinar às empresas do setor e também ao SUS. Disponível em < https://hazeshift.com.br/startups-telemedicina-healthtechss/.> Acesso em: 08 de novembro de 2021.

FURNIVAL AC. A participação dos usuários no desenvolvimento de sistemas de informação. Ciência da Informação 1995: 25: 2.

HORAN T, TULU B, HILTON B. Understanding Physician Use of Online Systems: An Empirical Assessment of an Electronic Disability Evaluation System, in EHealth Systems Diffusion and Use: The Innovation, the User and the USE IT Model, Ed. Schuring, R.W. and Spil, T.A.M., by Idea Group Inc. 2005.

INFANTOSI.A.F.C. Criação Bioengenharia no Brasil: entrevista: [junho.2001]. Entrevistadora: Ana Maria Antônio. 02 Fitas Cassetes.

KENSKI, V. M. Educação e tecnologias: o novo ritmo da informação (8a ed.). Campinas 2011, SP: Papirus.

LAPOINT L, LAMOTHE L, FORTIN J. The Dynamics of IT Adoption in a Major change Process in Healthcare Delivery. Proceedings of the 35th Hawaii International Conference on System Sciences, 2002.

LOBO, LUIZ CARLOS. Inteligência artificial, o Futuro da Medicina e a Educação Médica. Disponível em: $<$ https://www.scielo.br/j/rbem/a/PyRJrW4vzDhZKzZW47wddQy/?lang=pt\#>. Acesso em: 05 de novembro de 2021. 
MAGALHÃES, C. A. S. Análise da resistência médica à implantação de sistemas de registro eletrônico de saúde.[Analysis of Medical Resistance to the Introduction of Systems for Electronic Health Records]. 2006.

MUNDY D, CHADWICK DW. Eletronic transmission of prescriptions: towards realizing the dream. Int. J. Eletronic Healthcare 2004: 1.

MURARO, R.M. Os avanços tecnológicos e o futuro da humanidade: querendo ser Deus? Petrópolis (RJ):Vozes; 2009.

OLIVEIRA, S. M. Início da Bioengenharia na Usp e no Brasil: entrevista: [maio, 2003]. Entrevistadora: Ana Maria Antônio. 02 Fitas Cassetes.

PAIM, J.S. Vigilância da saúde: tendências de reorientação de modelos assistenciais para a promoção da saúde. In: Czeresnia D, Freitas CM.Promoção da saúde: conceitos, reflexões, tendências.Rio de Janeiro (RJ): Fiocruz; 2005. p.51930.

PARÉ G. Implementing clinical information systems: A multiple-case study within a US Hospital. Health Services Management Research 2002; 15: 71-02.

Programa de Engenharia Biomédica. COPPE/UFRJ. Disponível em: <http://www.peb.ufrj.br/noticias/RevistaBiomedica40Anos.PDF>. Acesso em: 06 de novembro de 2021.

PREGUER, C.M. Educação médica continuada à distância em endocrinologia e metabologia. Arq. Bras. Endocrinol Metab. 2005;49(4):584-95

TAN J. E-Health Care Information Systems: An Introduction for Students and Professionals. Jossey-Bass; 2005.

VERZUH, E. MBA Compacto gestão de projetos. Rio de Janeiro: Campus, 2000

RC: 106092

Disponível em: https://www.nucleodoconhecimento.com.br/tecnologia/tecnologias-namedicina 
Enviado: Novembro, 2021.

Aprovado: Fevereiro, 2022.

RC: 106092

Disponível em: https://www.nucleodoconhecimento.com.br/tecnologia/tecnologias-namedicina 